\title{
Dynamic Modeling and Identification of an Heterogeneously Actuated Underwater Manipulator Arm
}

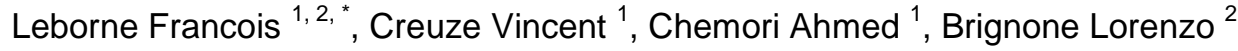 \\ ${ }^{1}$ Univ Montpellier, CNRS, LIRMM, 161 Rue Ada, F-34095 Montpellier 5, France. \\ ${ }^{2}$ Ifremer, Ctr Mediterranee, F-83507 La Seyne Sur Mer, France. \\ * Corresponding author : François Leborne, email address : francois.leborne@lirmm.fr
}

\begin{abstract}
:
This paper deals with the dynamic modeling and identification of an electrically driven underwater robot manipulator. The proposed study includes the dynamic modeling of the actuators of the arm as well as the identification of the parameters of the model. The proposed method deals with the specific case of heterogeneously actuated arms, namely arms with actuators behaving differently for each joint, being considered at the kinematic level. Indeed, we show how to estimate the arms parameters when some of their revolute joints are directly actuated by geared motors, while the others are actuated by linear actuators. A minimum set of identifiable parameters is determined, and adequate excitation trajectories are generated and used in the identification procedure. Real-time experimental validation on the manipulator arms of Ifremer's HROV (Hybrid Remotely Operated Vehicle) Ariane underwater vehicle demonstrates that the proposed method improves the estimation of the dynamic model.
\end{abstract}




\section{INTRODUCTION}

Manipulation tasks are required by several underwater applications, such as scientific research, oil and gas industry, or rescue. As a result, the manipulation of objects by means of an underwater vehicle has been addressed for several years, but until today most of the tasks are still performed by human operators remotely controlling the manipulator arms of the vehicle. The automation of a part of underwater manipulation tasks could greatly simplify the work of ROV pilots by allowing them to focus only on the mission's specific tasks requiring their expertise.

Autonomous underwater manipulation is a relatively new research topic, addressed by a few projects during the last two decades. The first project aiming at providing underwater dexterous manipulation capabilities was AMADEUS [1], [2]. In this project, two hydraulic manipulators were simultaneously controlled for manipulation tasks at depth up to 500 meters below sea surface thanks to model-based hybrid position/force controllers. The problem of controlling an UVMS (Underwater Vehicle-Manipulator System) has also been addressed in [3], where the controller also included the vehicle's dynamics. Other UVMS are described in [4], but all of them only feature joints whose motion in linearly related to the motion of the motors, which is usually expressed by the well-known $\boldsymbol{\tau}=\boldsymbol{B} \boldsymbol{u}$. In fact, in all these works, the dynamic behavior of the actuation system has either been neglected, or been shortly addressed in the case of hydraulic actuators. However, the modeling of electric actuators has been addressed by [5] and [6], where electric gear motors directly actuate the joints. These models involve dynamic parameters that need to be identified. In [7], to excite and identify the model's dynamic parameters, a method to generate specific trajectories is introduced. To estimate the values of the dynamic parameters, the recorded torque, position, velocity and acceleration are processed using the least mean square method, or other optimization methods as shown in [8]. Only a few parameters can actually be estimated, because of linear dependencies in the set of dynamic parameters [9]. This minimal set of linearly independent dynamic parameters can be determined analytically for the classical model of a manipulator [10]. However, when the dynamics of unusual actuators is considered, the minimal set of identifiable parameters is yet to be determined.

In this paper, we propose a new method to model and identify the dynamic parameters of an underwater manipulator. With respect to previous work, the first contribution consists in the inclusion of the actuator's dynamics, considering two different types of joints actuation. In fact, in our model, the revolute joints can either be directly actuated by a gear motor, or actuated by an electric linear actuator (Fig. 2). Then, we generate adequate identification trajectories to estimate our model's parameters. The paper also presents experimental results obtained with the manipulator arms of Ifremer's ${ }^{1}$ HROV Ariane. In section II, we describe the vehicle and its manipulators, and we derive their dynamic model, including the heterogeneous actuation. In section III, we propose a way to group together subsets of dynamic parameters, in order to obtain a minimum set of identifiable parameters. Finally, the whole method is experimentally validated in section IV.

\section{MODELING OF ARIANE'S MANIPULATOR ARMS}

\section{A. HROV Ariane and its manipulator arms}

The system considered for this work is Ifremer's HROV Ariane and its two electric manipulator arms. Ariane is an hybrid AUV/ROV system designed to operate up to 2500 meters deep. It measures $2.7 \mathrm{~m}$ in length, $1.2 \mathrm{~m}$ in width, $2.1 \mathrm{~m}$ in height and weighs a total of $1800 \mathrm{~kg}$ in its most complete configuration. As an hybrid system, it is designed to operate either as an ROV (Remotely Operated Vehicle) for remotely controlled underwater operations, or as an AUV (Autonomous Underwater Vehicle) for autonomous survey missions. The intervention tasks typically encountered for scientific use include collecting biological or geological samples. These tasks are performed by two manipulator arms

\footnotetext{
${ }^{1}$ Ifremer: French Research Institute for Exploitation of the Sea
} 
and exploit bespoke coring tools for sampling the seabed sediments, or biological live species such as gorgonian and corals.

Ariane's manipulator arms (Fig. 1) are manufactured by ECA Group and feature custom electronics and software developed by Ifremer. They both exclusively integrate revolute joints (four and six degrees of freedom, respectively) and a gripper. These electric manipulator arms are heterogeneously

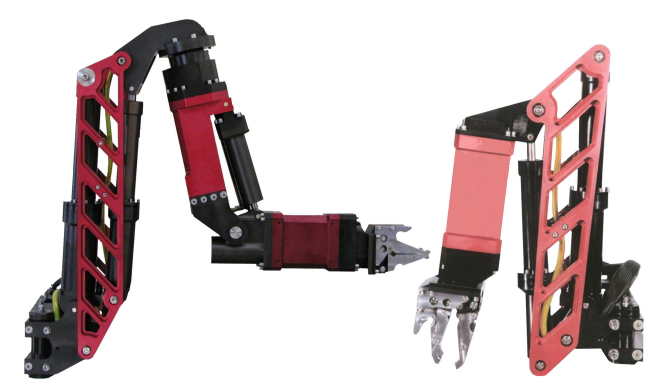

Fig. 1. Manipulator arms of Ariane: the 6-DOF (left) and 4-DOF (right).

actuated, since, depending on the joint, the motor is coupled with mechanical drive components implementing different actuation architectures:

- a gear motor directly actuating the joint, largely described and modeled in literature (see [5] for example)

- a gear motor coupled to a ball-screw-lever chain (i.e. an electric linear actuator)

To the authors knowledge, the later architecture, as presented in Fig. 2 with the notations introduced in table I, is specific to underwater manipulators and has not been studied from a dynamic point of view yet.

It is also worth noting that because of the low speed of each joint, the hydrodynamic effects can be neglected, and the non linearity of the actuator's behavior only is non negligible and therefore addressed here. This assumption is valid in our case, since we use low-speed manipulator arms, but may be wrong for faster underwater manipulators. It also allows us to identify the model in air.

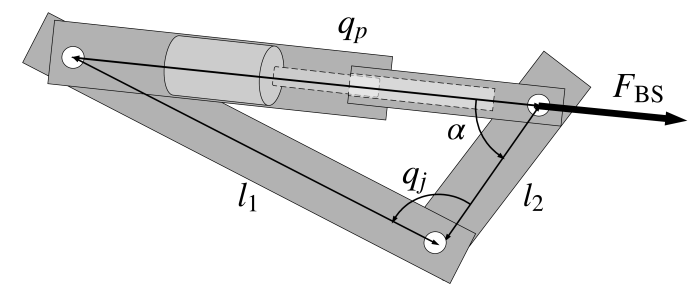

Fig. 2. Simplified drawing of an electric linear actuator (i.e. gear motorball-screw-lever) actuating a revolute joint. Most of the joints of Ariane's manipulator arms are actuated by this mechanism.

\section{B. Modeling of the electric actuators}

The purpose of this work is to derive the dynamic model of the manipulators, considering the input current of each motor as the input of the system and each joint coordinate
TABLE I

NOTATIONS USED IN THIS WORK

\begin{tabular}{c|l|c} 
Variable & Description & Unit \\
\hline$l_{1}, l_{2}$ & $\begin{array}{l}\text { lengths between the axis of the joint and each } \\
\text { end of the linear actuator }\end{array}$ & $\mathrm{m}$ \\
\hline$q_{p}$ & length of the cylinder & $\mathrm{m}$ \\
\hline$q_{j}$ & joint coordinate & $\mathrm{rad}$ \\
\hline$\alpha$ & $\begin{array}{l}\text { angle between the direction of } F_{\mathrm{BS}} \text { and the link } \\
\text { it is applied to }\end{array}$ & $\mathrm{rad}$ \\
\hline$F_{\mathrm{BS}}$ & force exerted by the cylinder on $L_{2}$ & $\mathrm{~N}$
\end{tabular}

as the output. In other words, we aim to improve the classical model of a manipulator as described in [11] by considering the actuators dynamics.

Our approach is based on two steps. First, we derive the classical model of a manipulator, in the following form:

$$
\boldsymbol{M}(\boldsymbol{q}) \ddot{\boldsymbol{q}}+\boldsymbol{N}(\boldsymbol{q}, \dot{\boldsymbol{q}})=\boldsymbol{\tau}
$$

where $\boldsymbol{q}$ is the vector of generalized joint coordinates of the system, $\boldsymbol{M}(\boldsymbol{q})$ is the inertia and masses matrix, $\boldsymbol{N}(\boldsymbol{q}, \dot{\boldsymbol{q}})$ is a stack vector that includes the non-inertial forces (Coriolis and friction) and the gravity and buoyancy term, and $\tau$ is the stack vector of the torques applied to the joints of the system. This part has been widely covered in literature, so we focus on the second step, consisting in completing the classical dynamic model with the dynamics of the actuators. In the case of Ariane's manipulators, we distinguish two kinds of actuators, which both have a different dynamic model. These models are derived as explained in the rest of this section.

In the case of a directly actuated joint (through a gear motor), the torque applied to the joint is directly the torque $\tau_{m}$ generated by the gear motor. As far as the modeling of such system is concerned, this is well-known and described in [5] for example and is expressed as follows:

$$
\tau_{m}=k_{T} i-r^{2}\left(J_{m} \ddot{q}_{m}+f_{v_{m}} \dot{q}_{m}+f_{s_{m}} \operatorname{sign}\left(\dot{q}_{m}\right)\right)
$$

where $i[\mathrm{~A}]$ is the input current, $k_{T}[\mathrm{Nm} / \mathrm{A}]$ is the torque constant of the motor, $r$ is the gearbox ratio, $\dot{q}_{m}[\mathrm{rad} / \mathrm{s}]$ is the angular velocity of the motor, $\ddot{q}_{m}$ is the angular acceleration of the motor, and $J_{m}, f_{v_{m}}$ and $f_{s_{m}}$ are the moment of inertia, viscous friction and dry friction coefficients of the gear motor, respectively.

In the case of a revolute joint actuated by a linear actuator, the rotation of the gear motor is not linearly transformed into the rotation of the joint. In order to model this transformation, we take apart the whole actuator into three subsystems: the electric gear motor, the ball-screw, and the connection between the tip of the actuator and arm's link. Then, our proposed process follows three steps:

- first, we write the kinematic equation of the subsystem

- then, we consider the inertia forces

- finally, we add the friction forces, modeled using both dry friction and viscous friction models

Since the modeling of the gear motor has already been covered (see (2)), we only describe the modeling of the 
ball-screw and the so-called lever, which converts the linear displacement into a rotational displacement.

1) Dynamic modeling of the ball-screw: $F_{\mathrm{BS}}$, the force applied by the linear actuator to the link of the manipulator, is expressed as:

$$
F_{\mathrm{BS}}=\frac{2 \pi}{p} \tau_{m}-I_{\mathrm{BS}} \ddot{q}_{p}-f_{s_{\mathrm{BS}}} \operatorname{sign}\left(\dot{q}_{p}\right)-f_{v_{\mathrm{BS}}} \dot{q}_{p}
$$

The ball-screw features a pitch $p$ and converts the torque $\tau_{m}$ produced by the gear motor into a linear force with a conversion factor $\frac{2 \pi}{p}$. We note $I_{\mathrm{BS}}$ the inertia of the translating parts, $f_{s_{\mathrm{BS}}}$ their dry friction coefficient and $f_{v_{\mathrm{BS}}}$ their viscous friction coefficient, $\dot{q}_{p}$ the linear velocity of the screw, and $\ddot{q}_{p}$ its linear acceleration.

We remark that the ball-screw system features components in linear and angular motion. These are characterized by differing inertia and friction models resulting into two differing viscous friction coefficients and two differing dry friction coefficients. However, the motion of the rotating parts is the same as the motion of the gear motor, so we can merge the dynamic parameters of the rotating parts of the ballscrew with the ones of the gear motor. This explains why the rotational motion does not explicitly appear in (3).

2) Mechanic modeling of the lever: The lever converts the force $F_{\mathrm{BS}}$ of the ball-screw into a torque $\tau_{l}$ applied to the joint with a conversion factor non-linearly dependent on the joint coordinate $q$ :

$$
\tau_{l}=l_{2} \sin (\alpha) F_{\mathrm{BS}}
$$

In this equation, we use a secondary angle, $\alpha$ as depicted on the Fig. 2, to simplify the expression of the conversion factor of the lever. It is straightforward that the value of the conversion factor is $l_{2} \sin \alpha$, with $l_{2}$ the length between the axis of the joint and the end of the linear actuator acting on the arm. There are no inertia forces nor frictions to take into consideration here, since they are already accounted in the classical model of the manipulator.

3) Dynamic modeling of the whole actuator: By merging (2), (3) and (4), we can finally write the model of the full actuator in the following form:

$$
\tau_{l}=k^{L}\left(q_{m}\right) i-m_{e q}^{L}\left(q_{m}, \ddot{q}_{m}\right)-f_{e q}^{L}\left(q_{m}, \dot{q}_{m}\right)
$$

with these notations:

$$
\begin{aligned}
k^{L}\left(q_{m}\right) & =\frac{2 \pi}{p} k_{T} l_{2} \sin (\alpha) \\
m_{e q}^{L}\left(q_{m}, \ddot{q}_{m}\right) & =l_{2}\left(\frac{2 \pi}{p} r^{2} J_{m}+p I_{B S}\right) \sin (\alpha) \ddot{q}_{m} \\
f_{e q}^{L}\left(q_{m}, \dot{q}_{m}\right) & =l_{2}\left(\frac{2 \pi}{p} r^{2} f_{v_{m}}+p f_{v_{p}}\right) \sin (\alpha) \dot{q}_{m} \\
& +l_{2}\left(\frac{2 \pi}{p} r^{2} f_{s_{m}}+p f_{s_{p}}\right) \sin (\alpha) \operatorname{sign}\left(\dot{q}_{m}\right)
\end{aligned}
$$

where the superscript $L$ refers to a joint actuated by a linear actuator.

\section{Dynamic model of the manipulator arm including actua- tors dynamics}

The model of the manipulator arm, considering the torque on each joint as the input of the system, is well-known and recalled in (1). In order to model the manipulator and its actuators dynamics, the next step is to replace $\tau$ with the dynamic model of the actuators producing each generalized torque of the system. If joint $j$ is a directly actuated joint, then $\tau_{j}=\tau_{m}$ (from (2)), else if joint $j$ is actuated by a linear actuator, then $\boldsymbol{\tau}_{j}=\tau_{l}$ (from (5)).

To ensure consistency of the model, we express, for each joint, the $q_{m}$ coordinate (related to the shaft of each motor) as a function of the corresponding generalized coordinate $q$, which is the joint coordinate used by the notation proposed by Khalil and Kleinfinger in [12]:

$$
\begin{aligned}
& \dot{q}_{m}=k_{1}(q) \dot{q} \\
& \ddot{q}_{m}=k_{1}(q) \ddot{q}+k_{2}(q, \dot{q})
\end{aligned}
$$

Then, we use this expression to write the model of each actuator using the generalized coordinates. Equation (7) also shows that the models of the actuators are linear in $\ddot{q}$, so it is possible to rewrite $\tau$ from (1) in the following form:

$$
\boldsymbol{\tau}=\boldsymbol{K}(\boldsymbol{q}) \boldsymbol{i}-\boldsymbol{M}_{\text {actuators }}(\boldsymbol{q}) \ddot{\boldsymbol{q}}-\boldsymbol{N}_{\text {actuators }}(\boldsymbol{q}, \dot{\boldsymbol{q}})
$$

where $\boldsymbol{K}(\boldsymbol{q})$ is a diagonal matrix, whose diagonal elements are:

$$
\boldsymbol{K}_{j, j}(\boldsymbol{q})= \begin{cases}k_{T}^{j} & \text { if joint } j \text { is direct } \\ \frac{2 \pi}{p^{j}} k_{T}^{j} l_{2}^{j} & \text { if joint } j \text { is levered }\end{cases}
$$

As a result, the classical model of a manipulator arm is rewritten to consider the input current of each motor as the input of the system:

$$
\boldsymbol{K}(\boldsymbol{q}) \boldsymbol{i}=\boldsymbol{M}^{\star}(\boldsymbol{q}) \ddot{\boldsymbol{q}}+\boldsymbol{N}^{\star}(\boldsymbol{q}, \dot{\boldsymbol{q}})
$$

by defining $M^{\star}$ and $N^{\star}$ using $M$ and $N$ from (1):

$$
\begin{aligned}
M^{\star}(\boldsymbol{q}) & =\boldsymbol{M}(\boldsymbol{q})+\boldsymbol{M}_{\text {actuators }}(\boldsymbol{q}) \\
\boldsymbol{N}^{\star}(\boldsymbol{q}, \dot{\boldsymbol{q}}) & =\boldsymbol{N}(\boldsymbol{q}, \dot{\boldsymbol{q}})+\boldsymbol{N}_{\text {actuators }}(\boldsymbol{q}, \dot{\boldsymbol{q}})
\end{aligned}
$$

In (10) and for the rest of this article, the $\star$ superscript indicates the objects taking into account the dynamics of the actuators.

\section{Manipulator and actuators model identification}

It is well-known ([13], [10]) that the dynamic identification model of a manipulator arm is expressed by $\boldsymbol{\Phi}(\boldsymbol{q}, \dot{\boldsymbol{q}}, \ddot{\boldsymbol{q}}) \boldsymbol{\theta}=\boldsymbol{\tau}$, where $\boldsymbol{\theta}$ is the stack vector of the dynamic parameters of the model to be identified, and $\boldsymbol{\Phi}$ is the associated regressor. In order to derive this model for the manipulator arm including its actuators' dynamics, we replace $\tau$ by its expression given in (8):

$$
\boldsymbol{\Phi}(\boldsymbol{q}, \dot{\boldsymbol{q}}, \ddot{\boldsymbol{q}}) \boldsymbol{\theta}+\boldsymbol{M}_{\text {actuators }}(\boldsymbol{q}) \ddot{\boldsymbol{q}}+\boldsymbol{N}_{\text {actuators }}(\boldsymbol{q}, \dot{\boldsymbol{q}})=\boldsymbol{K}(\boldsymbol{q}) \boldsymbol{i}
$$

From (2) and (5), it can be seen that the actuators' model is linear in its dynamic parameters, so there exists a regressor 
$\boldsymbol{\Phi}_{\text {actuators }}$ and a vector of dynamic parameters $\boldsymbol{\theta}_{\text {actuators }}$ such that (12) can be rewritten:

$$
\boldsymbol{\Phi}(\boldsymbol{q}, \dot{\boldsymbol{q}}, \ddot{\boldsymbol{q}}) \boldsymbol{\theta}+\boldsymbol{\Phi}_{\text {actuators }}(\boldsymbol{q}, \dot{\boldsymbol{q}}, \ddot{\boldsymbol{q}}) \boldsymbol{\theta}_{\text {actuators }}=\boldsymbol{K}(\boldsymbol{q}) \boldsymbol{i}
$$

This means that we can define the regressor $\boldsymbol{\Phi}^{\star}$ and the stack vector $\boldsymbol{\theta}^{\star}$ of the unknown dynamic parameters of the model as:

$$
\begin{aligned}
\boldsymbol{\Phi}^{\star} & =\boldsymbol{K}^{-1}(\boldsymbol{q})\left[\boldsymbol{\Phi}, \boldsymbol{\Phi}_{\text {actuators }}\right] \\
\boldsymbol{\theta}^{\star} & =\left[\boldsymbol{\theta}^{T}, \boldsymbol{\theta}_{\text {actuators }}^{T}\right]^{T}
\end{aligned}
$$

so that the dynamic identification model of the manipulator arm, including its actuators' dynamics, becomes:

$$
\boldsymbol{\Phi}^{\star}(\boldsymbol{q}, \dot{\boldsymbol{q}}, \ddot{q}) \boldsymbol{\theta}^{\star}=\boldsymbol{i}
$$

in which $i$ is the stack vector of the current inputs of the system. We remark that $\boldsymbol{K}(\boldsymbol{q})$ is invertible by construction, as shown in (9).

\section{ESTIMATION OF THE BASE DYNAMIC PARAMETERS OF THE MODEL}

The previously derived model features a number a parameters whose value has to be determined in order for the model to represent the real system. Not all of these parameters can be estimated; only the so-called base parameters, which are the minimum set of parameters needed to compute the dynamic model [9], can be estimated. In this work, we use the OpenSymoro software tool to obtain the base parameters of the classical model of a manipulator arm, without the actuators dynamics. Then, we determine the set of base parameters of the actuators model. Finally, we group both sets of parameters to obtain the complete set of base dynamic parameters of the full model.

\section{A. Base parameters related to the actuators directly driving the joint}

In (2), it can be easily shown that the set $\left(J_{m}, f_{v_{m}}, f_{s_{m}}\right)$ is minimal, since there is no linear relation between $\ddot{q}_{m}(t)$, $\dot{q}_{m}(t)$, and $\operatorname{sign}\left(\dot{q}_{m}(t)\right)$. Therefore there are no dynamic parameters to merge in the model of the actuators directly driving the joints.

\section{B. Base parameters related to lever configured actuators}

Generally speaking, it is possible to generate a reference trajectory $q(t)$ such that there is no linear relation between $\ddot{q}(t), \dot{q}(t)$, and $\operatorname{sign}(\dot{q}(t))$, so inertia, fluid friction coefficient and viscous friction coefficient don't have to be grouped together. However, an affine relation exists between $q_{m}(t)$ and $q_{p}(t)$, expressed as $q_{m}(t)=p q_{p}(t)-q_{p_{\min }}, p$ being the pitch of the ball-screw. This leads to a linear relation between $\ddot{q}_{m}(t)$ and $\ddot{q}_{p}(t), \dot{q}_{m}(t)$ and $\dot{q}_{p}(t)$, and $\operatorname{sign}\left(\dot{q}_{m}(t)\right)$ and $\operatorname{sign}\left(\dot{q}_{p}(t)\right)$. This is why in (5), $\ddot{q}_{m}(t)$ is factorized, which means that the inertias of the gear motor and the ball-screw cannot be decoupled and therefore have to be merged into a single base parameter $J_{\text {actuator }}$, with the following expression:

$$
J_{\text {actuator }}=l_{2}\left(\frac{2 \pi}{p} r^{2} J_{m}+p I_{B S}\right)
$$

Similarly, we define the base parameters $f_{s_{\text {actuator }}}$ and $f_{v_{\text {actuator }}}$ as:

$$
\begin{aligned}
& f_{s_{\text {actuator }}}=l_{2}\left(\frac{2 \pi}{p} r^{2} f_{s_{m}}+p f_{s_{p}}\right) \\
& f_{v_{\text {actuator }}}=l_{2}\left(\frac{2 \pi}{p} r^{2} f_{v_{m}}+p f_{v_{p}}\right)
\end{aligned}
$$

\section{Simplification of the torque expression}

It can be seen in (2) and (5) that for each type of actuator, the torque applied to the joint is proportional to the input current $i$ (directly actuated joint) or to $\sin (\alpha) i$ (lever actuated joint). The involved proportionality coefficients can be merged with the dynamic base parameters, due to the fact that the dynamic model is linear in these coefficients. This avoids the need of a precise knowledge of each joint's $k_{T}$, $l_{2}$ and $p$ parameters, even though the last two are required for the derivation of the kinematic model.

\section{Base parameters of the whole system}

Finally, each actuator type has a set of three dynamic base parameters: an inertia, a viscous friction coefficient, and a dry friction coefficient. Then, the base parameters of the whole system are the union of the base parameters of each actuator, and of the base parameters retrieved from the classical manipulator arm's model.

\section{E. Estimation of the parameters}

We define the vector $\boldsymbol{\theta}^{\star}$ by stacking the dynamic base parameters, and it verifies $\boldsymbol{\Phi}^{\star}(\boldsymbol{q}, \dot{\boldsymbol{q}}, \ddot{\boldsymbol{q}}) \boldsymbol{\theta}^{\star}=\boldsymbol{i}$, as presented in section II-D. In order to improve the quality of the estimation, we record the state of the manipulator arm as it follows an excitation reference trajectory and use this data to create the augmented system $\boldsymbol{F}(\boldsymbol{q}, \dot{\boldsymbol{q}}, \ddot{\boldsymbol{q}}) \boldsymbol{\theta}^{\star}=\boldsymbol{b}$, where

$$
\begin{gathered}
\boldsymbol{F}=\left[\begin{array}{c}
\boldsymbol{\Phi}^{\star}(\boldsymbol{q}(0), \dot{\boldsymbol{q}}(0), \ddot{\boldsymbol{q}}(0)) \\
\vdots \\
\boldsymbol{\Phi}^{\star}(\boldsymbol{q}(N), \dot{\boldsymbol{q}}(N), \ddot{\boldsymbol{q}}(N))
\end{array}\right] \\
\boldsymbol{b}=[\boldsymbol{i}(0) \cdots \boldsymbol{i}(N)]^{T}
\end{gathered}
$$

The excitation reference trajectory $\left(\boldsymbol{q}_{r}(t), \dot{\boldsymbol{q}}_{r}(t), \ddot{\boldsymbol{q}}_{r}(t)\right)$ is computed offline, with the aim of minimizing the condition number of the observation matrix $\boldsymbol{F}\left(\boldsymbol{q}_{r}(t), \dot{\boldsymbol{q}}_{r}(t), \ddot{\boldsymbol{q}}_{r}(t)\right)$. The reader can find more details about the estimation of dynamic parameters in [14] and about the computation of an excitation trajectory in [7].

\section{EXPERIMENTAL VALIDATION}

The augmented model of the manipulator including the dynamics of the actuators, as well as the estimation process of the dynamic parameters, have been validated using the 4-DOF manipulator arm of Ifremer's HROV Ariane. The Khalil-Kleinfinger parameters [12] and the lengths related to each lever actuated joint of this manipulator arm are presented in table II.

Although the considered manipulator arm has four degrees of freedom, only three will be considered in this work, 
TABLE II

GEOMETRIC PARAMETERS OF THE 4-DOF MANIPULATOR ARM

\begin{tabular}{c|r|r|r|r|r} 
Joint & $d[\mathrm{~m}]$ & $r[\mathrm{~m}]$ & $\alpha[\mathrm{rad}]$ & $l_{1}[\mathrm{~m}]$ & $l_{2}[\mathrm{~m}]$ \\
\hline 1 & 0 & 0 & 0 & 0.323 & 0.058 \\
\hline 2 & 0.116 & 0 & $\frac{\pi}{2}$ & 0.073 & 0.537 \\
\hline 3 & 0.443 & 0 & 0 & 0.489 & 0.054 \\
\hline 4 & -0.1 & 0.436 & $-\frac{\pi}{2}$ & - & -
\end{tabular}

because the joint 4 , which rotates the gripper, is not equipped with a position sensor, so its joint coordinate is not accurately known during the experiments. This joint remains blocked during the whole experiment.

\section{A. Derivation of the identification model of the manipulator}

We derive the dynamic identification model (DIM) of the arm using OpenSymoro, and we complete it with the DIM of the actuators, derived using the symbolic toolbox of MATLAB ${ }^{\circledR}$. The result of this process is the DIM $\Phi^{\star}$ of the arm and its actuators, as described in (14).

\section{B. Computation and tracking of the reference excitation trajectory}

The reference excitation trajectory is computed using the method described in [7]. The number of degrees of freedom $n$ of the arm is 3 , the number of harmonics $N$ for each trajectory is set to 5 , since this offers a viable compromise between computation time and the complexity of the trajectory. The optimization constraints are defined for each joint, as presented in table III. Because of the non-linearity of the transformation of the rotation of the motors in the rotation of the joints, we choose to generate the reference trajectory in motors space. This means that the generated trajectory $\boldsymbol{q}(t)$ represents a number of motors steps (i.e. signal from the hall effect sensor used for electronic commutation) counted from a zero position. For the same reason, the constraints, which correspond to physical limitations of the motors, are not given in radians, $\mathrm{rad} / \mathrm{s}$, and $\mathrm{rad} / \mathrm{s}^{2}$ but in increments, inc/s, and inc $/ \mathrm{s}^{2}$, respectively. However, for ease of understanding, we also give the equivalent of the motors constraints in the joints space in table III.

TABLE III

CONSTRAINTS OF THE OPTIMIZATION PROBLEM

\begin{tabular}{l|c|r|r|r} 
& Unit & Joint 1 & Joint 2 & Joint 3 \\
\hline $\boldsymbol{q}_{m}$ & {$[$ inc $]$} & {$[0 ; 20850]$} & {$[0 ; 6810]$} & {$[0 ; 26750]$} \\
\hline$\dot{\boldsymbol{q}}_{m, \max }$ & {$[\mathrm{inc} / \mathrm{s}]$} & 2000 & 600 & 2000 \\
\hline$\ddot{\boldsymbol{q}}_{m, \max }$ & {$\left[\mathrm{inc} / \mathrm{s}^{2}\right]$} & 20000 & 6000 & 20000 \\
\hline $\boldsymbol{q}$ & $\left.{ }^{\circ}\right]$ & {$[-90 ; 32]$} & {$[7 ; 94]$} & {$[110 ; 244]$} \\
\hline$\dot{\boldsymbol{q}}_{\max }$ & {$\left[{ }^{\mathrm{o}} / \mathrm{s}\right]$} & 28 & 9 & 25
\end{tabular}

The optimization of the trajectory is done using MATLAB $^{\circledR}$ Toolbox constr, and the interior-point method [15]. Because of the existence of many local minima, the solver is initialized several times with random values, until it converges to a solution for which the condition of the observation matrix is less than an arbitrary threshold. Once an excitation trajectory is obtained, it is set as a reference trajectory to be tracked by the real system. One of the resulting reference trajectories is shown in Fig.3. The sampling frequency is $10 \mathrm{~Hz}$, so the duration of a period is 80 seconds.

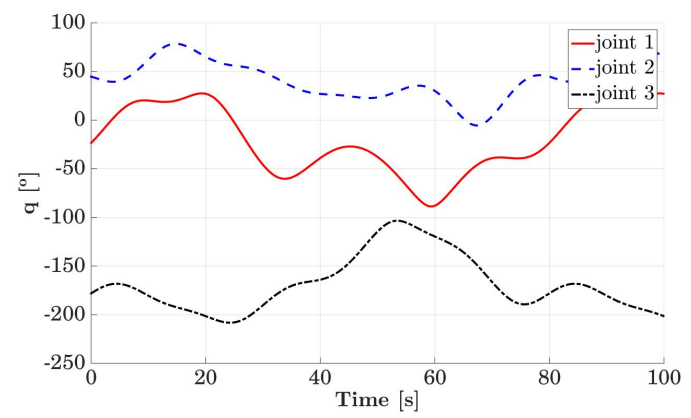

Fig. 3. A reference excitation trajectory for the three controlled joints, that satisfies the constraints given in table III

To track the reference trajectory, each generalized coordinate of the manipulator arm is controlled by three PI (Proportional Integral) controllers. In fact, a PI current regulator, a PI velocity regulator and a PI position regulator are cascaded. The current regulation and the velocity tracking are realized by built-in PI controllers of ELMO Whistle ${ }^{\circledR}$ motor drivers. The reference position tracking is done by a custom ROS node and during the tracking of the reference trajectory, we record the position of the motors and their input currents in a ROS bag. All the data is provided and timestamped by the motor drivers, which avoids any real-time issues.

\section{Identification of the dynamic parameters}

The data recorded during the tracking of the excitation trajectory is exported to MATLAB ${ }^{\circledR}$ to create the augmented observation matrix $\boldsymbol{F}$ and the augmented inputs vector $\boldsymbol{b}$, as presented in (18). The estimated vector $\hat{\boldsymbol{\theta}}^{\star}$ of the dynamic parameters is obtained by solving $\boldsymbol{F}(\boldsymbol{q}, \dot{\boldsymbol{q}}, \ddot{\boldsymbol{q}}) \boldsymbol{\theta}^{\star}=\boldsymbol{b}$ with the method of least-squares:

$$
\hat{\boldsymbol{\theta}}^{\star}=\boldsymbol{F}^{+}(\boldsymbol{q}, \dot{\boldsymbol{q}}, \ddot{\boldsymbol{q}}) \boldsymbol{b}
$$

$\boldsymbol{F}^{+}$being the Moore-Penrose pseudo-inverse of $\boldsymbol{F}$.

\section{Validation of the model and its estimated parameters}

To validate the estimated parameters, we use them to estimate the input currents $\hat{b}$ related to the tracking of a validation trajectory:

$$
\hat{\boldsymbol{b}}=\boldsymbol{F}(\boldsymbol{q}, \dot{\boldsymbol{q}}, \ddot{\boldsymbol{q}}) \hat{\boldsymbol{\theta}}^{\star}
$$

Then, we compare $\hat{b}$ to the actual input currents $\boldsymbol{b}$, recorded while the real system is tracking the validation trajectory.

The recorded position is first filtered in order to remove noise, using a third order Butterworth filter with a normalized cutoff frequency of 0.1 . However, the recorded current is not filtered, since its filtering would affect to observability of the effect of the dry friction. 
The estimated input currents obtained in this experiment are presented and compared to the real input currents in Fig. 4. In order to quantitatively compare the two models,

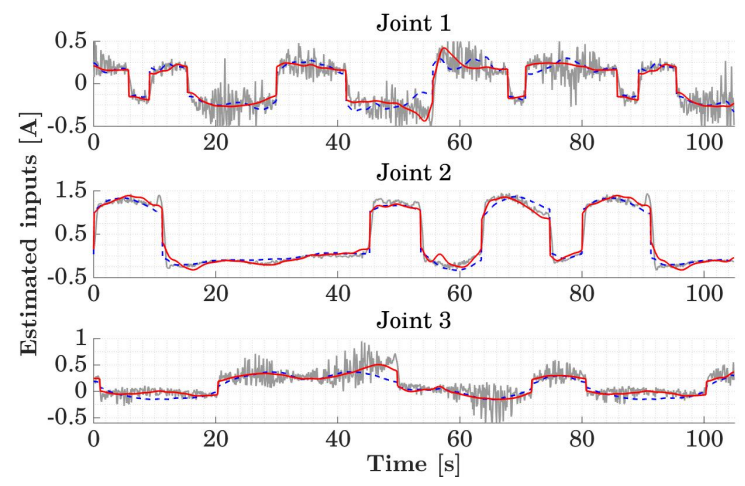

Fig. 4. Estimated input currents. The real current (solid gray line) is compared to the estimated input current, without (blue dashed line) and with (red solid line) actuators' dynamics.

the root mean square error (RMSE) of the estimated inputs for each joint is computed:

$$
\operatorname{RMSE}(\hat{\boldsymbol{b}})=\sqrt{\mathrm{E}\left((\hat{\boldsymbol{b}}-\boldsymbol{b})^{2}\right)}
$$

The value of the RMSE in each case is given in table IV.

TABLE IV

ROOT MEAN SQUARE ERROR OF THE ESTIMATION

\begin{tabular}{c|c|c|c} 
& Joint 1 & Joint 2 & Joint 3 \\
\hline without actuators dynamics $[\mathrm{A}]$ & 0.129 & 0.154 & 0.152 \\
\hline with actuators dynamics $[\mathrm{A}]$ & 0.117 & 0.128 & 0.122 \\
\hline improvement [\%] & 9.80 & 16.8 & 19.9
\end{tabular}

\section{E. Discussion}

Fig. 4 and table IV show that the consideration of the actuators' dynamics brings an improvement to the model. However, this improvement is not as good as we could expect from the simulation. Two particular features of our hardware implementation may explain this:

- the measure of the current given by the motor drivers is noisy, but most importantly, the built-in current regulators are unable to provide a stable current. The measured motion of the arm does not reflect this permanently oscillating current as it is filtered out by the mechanics of the manipulator;

- unmodeled flexibility and backlash affect the observability of the arm behavior over very short periods of time.

Consequently it is not possible to perfectly map the measured current to the actual behavior of the arm.

We also observe a difference between the RMSE of joint 1 on one hand, and the one of joints 2 and 3 on the other hand. This is due to joint 1's rotation axis being vertical, while joints 2 and 3's axes are horizontal, and thus both have to compensate for gravity effects, which results in a greater static error.

\section{CONCLUSION AND FUTURE WORK}

In this article, we have described an original method to model heterogeneously actuated underwater manipulator arms, with an emphasis on the properties of an electric linear actuator. The base dynamic parameters of this model have been determined and used to obtain the dynamic identification model of this type of actuators, which has been subsequently used to improve the dynamic model of a manipulator arm. The proposed model and the estimation of its parameters has been validated through experiments, using the 4-DOF electric manipulator arm fitted to Ifremer's HROV Ariane. Future work will exploit the obtained model to accurately simulate and control the arm in manipulation tasks, aiming to obtain better reliability and increased automation of scientific sampling tasks (biological and sediment).

\section{ACKNOWLEDGEMENT}

This work is jointly supported by Ifremer and Labex NUMEV (Grant AAP2014-2-04).

\section{REFERENCES}

[1] D. M. Lane, J. B. C. Davies, G. Robinson, D. J. O. Brien, J. Sneddon, E. Seaton, and A. Elfstrom, "The AMADEUS Dextrous Subsea Hand : Design , Modeling, and Sensor Processing," IEEE Journal of Oceanic Engineering, vol. 24, no. 1, pp. 96-111, 1999.

[2] M. W. Dunnigan, D. M. Lane, A. C. Clegg, and I. Edwards, "Hybrid position / force control of a hydraulic underwater manipulator," in IEEE Proc. Control Theory Appl., vol. 143, no. 2, 1996, pp. 145-151.

[3] Y. Cui and N. Sarkar, "A Unified Force Control Approach to Autonomous Underwater Manipulation," in IEEE International Conference on Robotics and Automation, 2000, pp. 1263-1268.

[4] G. Antonelli, "Underwater Robots." Springer International Publishing, 2014, ch. 1, pp. 1-22.

[5] R. Kelly, V. Davila, and J. Perez, "Control of robot manipulators in joint space." Springer, 2006, ch. 3, pp. 59-94.

[6] B. Siciliano, L. Sciavicco, L. Villani, and G. Oriolo, "Robotics: Modelling, Planning and Control." Springer, 2009, ch. 7, pp. 247302.

[7] J. Swevers, C. Ganseman, D. Bilgin Tükel, J. De Schutter, and H. Van Brüssel, "Optimal robot excitation and identification," IEEE Transactions on Robotics and Automation, vol. 13, no. 5, pp. 730-740, 1997.

[8] M. Gautier and P. Poignet, "Extended Kalman filtering and weighted least squares dynamic identification of robot," Control Engineering Practice, vol. 9, no. 12, pp. 1361-1372, 2001.

[9] M. Gautier and W. Khalil, "On the Identification of the Inertial Parameters of Robots," in IEEE Conference on Decision and Control, no. December, 1988, pp. 2264-2269.

[10] H. Mayeda, K. Yoshida, and K. Osuka, "Base Parameters of Manipulator Dynamic Models," IEEE Transactions on Robotics and Automation, vol. 6, no. 3, pp. 312-321, 1990.

[11] M. W. Spong, S. Hutchinson, and M. Vidyasagar, "Robot Dynamics and Control." Wiley, 2004, ch. 9, pp. 187-224.

[12] W. Khalil and J. Kleinfinger, "A new geometric notation for open and closed-loop robots," in Proceedings. 1986 IEEE International Conference on Robotics and Automation, vol. 3, no. I, 1986, pp. 11741179.

[13] M. Gautier and W. Khalil, "Direct calculation of minimum set of inertial parameters of serial robots," IEEE Transactions on Robotics and Automation, vol. 6, no. 3, pp. 368-373, Jun 1990.

[14] M. Gautier, "Optimal Motion Planning for Robot's Inertial Parameters Identification," in IEEE Conference on Decision and Control, 1992, pp. 70-73.

[15] R. A. Waltz, J. L. Morales, J. Nocedal, and D. Orban, "An interior algorithm for nonlinear optimization that combines line search and trust region steps," Mathematical Programming, vol. 107, no. 3, pp. 391-408, 2006. 